\title{
DISEÑO METODOLÓGICO PARA LA VALORACIÓN ECONÓMICA DE LOS SERVICIOS AMBIENTALES DE LA RESERVA ECOLÓGICA "HOLA VIDA"
}

José Gavidia B. ${ }^{1}$, Teresa Palacios C. ${ }^{2}$, Carmen Yauri B. ${ }^{3}$

\section{RESUMEN}

Los servicios ambientales que presta un bosque son incalculables y carecen de un mercado, es decir, no tienen un valor económico relacionado con cada uno de ellos, por tal motivo, son explotados de manera incontrolada sin prever las consecuencias que ello conlleva.

La importancia de establecer un valor económico a los servicios ambientales provenientes de los bosques, no solo radica en representar en cifras monetarias cuánto significarían las pérdidas de uno u otro recurso natural; sino más bien, representar la necesidad de mantener el equilibrio ecológico con respecto al cuidado y manejo de los recursos naturales, mediante la implementación de medidas que contribuyan al logro de un desarrollo sostenible que permita el balance entre la actividad económica y el desarrollo de la sociedad.

En Ecuador existen muy pocos estudios sobre la valoración de los servicios ambientales en Reservas Ecológicas; es por ello que la presente investigación sugiere un valor económico representativo a los principales bienes y servicios ambientales presentes en la Reserva Ecológica "Hola Vida", a través de métodos que incluyen la valoración contingente, costo de viaje, valoración basada en costes y costo de oportunidad.

La propuesta metodológica para la valoración de servicios ambientales, concentra elementos para obtener resultados relevantes, como parte de un mecanismo que permita encontrar el

1 Ministerio del Interior, Comandancia General de Policía Nacional, Quito, Ecuador (jlg_luis@yahoo.com).

2 Universidad Central del Ecuador, Facultad de Ingeniería, Minas, Petróleos y Ambiental, Quito, Ecuador (tere saalejandrap3@gmail.com).

3 Ministerio del Ambiente, Facilitadora socio-ambiental, Quito, Ecuador (c.yauri.facilitador.mae@gmail.com). 
valor de los principales bienes y servicios ambientales evaluados y a su vez, promuevan una concienciación ambiental dentro la sociedad que visita este importante recurso natural.

Palabras clave: bienes y servicios ambientales, Reserva Ecológica, valoración económica, ecosistemas, economía ambiental, recursos naturales, equilibrio ecológico.

\section{ABSTRACT}

The environmental services provided by a forest are incalculable and lack of a market, it does not have an economic value related to each of them, for that reason, they are exploited in an uncontrolled manner without considering the consequences that such action entails.

The importance of establishing an economic value to environmental services from forests lies not only in representing in monetary figures how much the loss of any natural resoucer would mean, but rather represent the need to maintain the Ecological balance regarding the care and management of natural resources by implementing measures that would contribute to achieving sustainable development that the balance between economic activity and the development of society allow.

In Ecuador there are very few studies on environmental valuation services in ecological reserves; it is for this reason that the current research suggests an economic value representative to the main environmental goods and services existing in the Ecological Reserve "Hola Vida," through methods that include the contingent valuation, cost of travel, valuation based on costs and opportunity cost.

The methodology for the valuation of environmental services focuses to obtain precise data elements as part of a mechanism to find the value of the main environmental goods and services evaluated and in turn, promote environmental awareness within society that visit this important natural resource.

Key words: environmental goods and services, Ecological Reserve, economic valuation, ecosystems, environmental economy, natural resources, ecological balance.

\section{INTRODUCCIÓN}

Los recursos naturales son parte fundamental en el progreso y desarrollo del ser humano, su agotamiento, dete- rioro y sus efectos no son claros y al no existir un mercado donde los bienes ambientales puedan ser intercambiados, no 
es posible obtener una curva de demanda; ello ha motivado el desarrollo de distintas técnicas para estimar sus beneficios o para valorar el daño que produce un deterioro ambiental, cuantificando hasta donde está dispuesto a sacrificar la sociedad en pos de una mejor calidad de vida (Gorfinkiel D, 1999).

El comportamiento actual de la sociedad en cuanto a la sobreexplotación de recursos naturales, ha generado cambios significativos sobre los diferentes ecosistemas agotando su capacidad de resistencia y poniendo en peligro la biodiversidad, lo cual, a su vez, ha provocado la extinción de numerosas especies a un ritmo acelerado, con graves consecuencias sobre la biósfera.

Este proceso de cambio, se evidencia en la cantidad y calidad de servicios y bienes ambientales que se obtienen en la actualidad.

"Hola Vida" es una Reserva Ecológica, ubicada al suroeste de la provincia de Pastaza, a 850 metros sobre el nivel del mar y cubre una superficie de 2,25 $\mathrm{km}^{2}$ (225 hectáreas) lo que representa el $0,04 \%$ del territorio de la parroquia Pomona. Desafortunadamente, la Reserva no cuenta con un análisis económico de los servicios ambientales que presta por su existencia a la sociedad. La falta de cuantificación del valor ambiental que posee un determinado lugar, impide que los recursos ambientales y los ecosistemas sean gestionados de forma óptima; además, cabe señalar que en economía los bienes que tienen un precio nulo se pueden consumir infinitamente, situación que propicia el inadecuado manejo de los recursos naturales; bajo este contexto, se prevé la necesidad de una valoración económica que nos permita dar a conocer a la sociedad, el costo ambiental que implica la pérdida de ecosistemas, de acuerdo con su grado de agotamiento y deterioro, así como, los efectos que ello genera sobre los niveles de producción, la salud humana o el bienestar social.

Los ecosistemas son dinámicos y se caracterizan por su complejo conjunto de plantas, animales y comunidades de microorganismos que conviven en un ambiente e interactúan como una unidad funcional. Los bienes y servicios del ecosistema son los productos de las funciones y atributos del mismo; $y$ desde el punto de vista antropocéntrico, estas funciones solo se convierten en servicios, una vez que los humanos las reconocen como parte de su sistema social y generan valores.

La economía ambiental busca cuantificar, en términos monetarios, los bienes y servicios ambientales sobre el entorno de las actividades económicas humanas, por lo que se precisa diseñar una metodología que permita valorar 
servicios ambientales no evaluados dentro de la Reserva Ecológica "Hola Vida", para resaltar el valor ecológico y cultural; y a su vez, establecer medidas de con- servación y manejo que garanticen la existencia de los recursos naturales para las futuras generaciones.

\section{OBJETIVOS}

- Diseñar una metodología que permita valorar servicios ambientales no evaluados dentro de la Reserva Ecológica "Hola Vida", para resaltar el valor ecológico y cultural.

- Realizar el levantamiento de línea base de la Reserva.

- Identificar los principales servicios ambientales potencialmente valorables.

- Determinar los costos ambienta- les asociados a cada uno de los servicios identificados.

- Determinar los beneficios de la valoración económica de los principales servicios ambientales.

- Proponer alternativas o mecanismos de sostenibilidad para la provisión de los servicios ambientales dentro de la reserva, desde el punto de vista económico y ecológico.

\section{METODOLOGÍA}

El área de estudio comprende la delimitación de la Reserva en cuadrantes, su tamaño se definió en base al FIA (Forest Inventory and Analysis) que muestrea parcelas de una hectárea (aproximadamente 2.5 acres), con el fin de garantizar la cobertura y calidad de la información a procesar, al tratarse de bosques (McRoberts, Tomppo, \& http:, 2015).

Los puntos principales de estudio fueron definidos a través de un muestreo sistemático que utiliza una cuadrí- cula fija o una matriz, para asignar parcelas en un patrón regular de 1 hectárea.

Según el tamaño de la muestra debe estar definida en función de la intensidad de muestreo, que establece los parámetros: área de bosque, área de parcela, intensidad de muestreo 2 \%, y número de parcelas.

El procedimiento para definir el número de parcelas consiste en lo siguiente: 
- Héctareas de bosque $(A)=225$ hectáreas

- Área de parcela (a) $=1$ hectárea

- Intensidad de muestreo requerida (i) = $2 \%$
- Número de hectáreas $(\mathrm{N})=225$

$$
\mathrm{n}=\mathrm{N} * \mathrm{i}
$$$$
n=225 * 0.02
$$

$\mathrm{n}=5$ parcelas

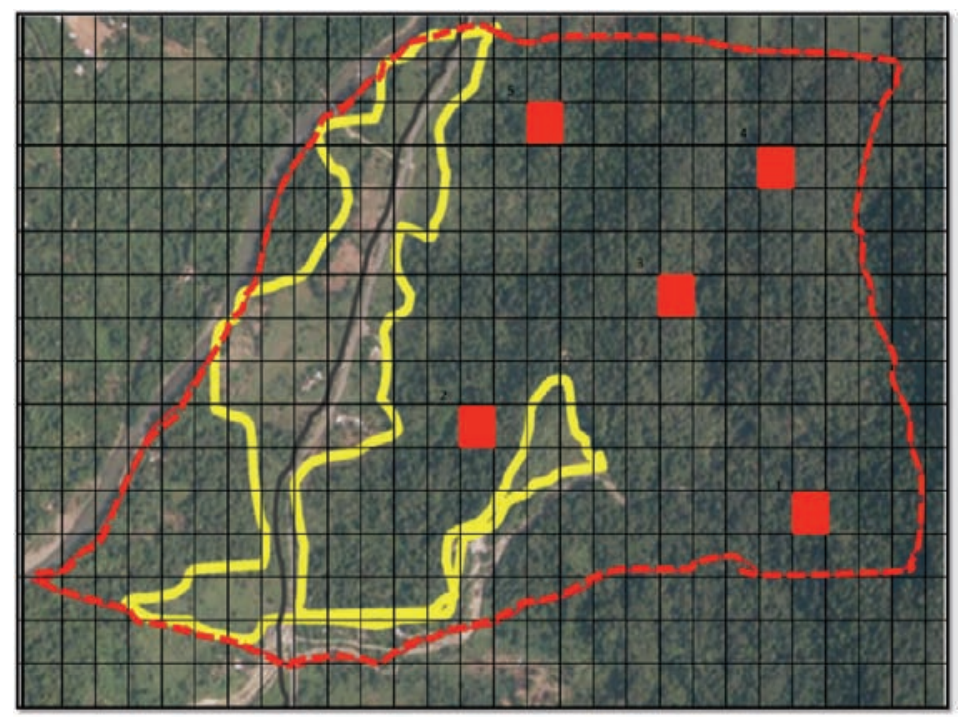

Figura 1. Sitios de muestreo

Se definieron 5 parcelas de 1 hectárea, las cuales fueron una muestra representativa para un bosque con características homogéneas, donde el diámetro a la altura del pecho de las especies boscosas, es superior a $15 \mathrm{~cm}$.

Para el desarrollo del siguiente trabajo se emplearon dos metodologías:
Metodología para la valoración de servicios ambientales: concentra elementos para mejorar los resultados y obtener datos más precisos, como un mecanismo que permita encontrar el valor de los bienes y servicios ambientales en la Reserva Ecológica "Hola Vida".

Para valorar las funciones ecológicas, en términos de bienes y servicios, en la reserva se han estimado cuatro fases: 
- Fase I: Identificación de bienes y servicios generados por la Reserva Ecológica Hola vida.

- Fase II: Jerarquización de los bienes y servicios con potencial para ser valorados económicamente.

- Fase III: Selección de los principales bienes y servicios ambientales a valorar.

- Fase IV: Determinación del valor económico de los bienes y servicios seleccionados.

\section{Metodología de Valoración Con-}

tingente: se ha convertido en una herramienta cada vez más popular para estimar cambios en el bienestar de las personas, especialmente cuando estos cambios involucran bienes no mercadeables cómo la naturaleza; se realizó con ayuda de un sistema informático software EViews 8, que es un programa sencillo para el análisis de datos econométricos, en este caso se utiliza, para conocer la disposición a pagar de las personas por conservar la reserva. Los resultados fueron analizados sobre una base de 300 individuos encuestados en la Reserva Ecológica "Hola Vida" (HANEMANN, 1984) (DUCCI, 1988) y (ARDILA, 1993).

El estudio consideró las siguientes variables:
- PRSI: Variable dependiente de naturaleza binaria. Representa la probabilidad de responder SI (=1) a la Disposición a Pagar-DAPag o No $(=0)$ de otra forma.

- PH: Precio hipotético ofertado sobre el que compara el entrevistado su disposición a pagar-DAPag.

- INGr: Ingreso familiar mensual.

- EDAD: Del jefe de familia o grupo. Toma el valor de 1 si está dentro de lo que se considera PEA y 0 de cualquier otra forma.

- EDUC: Educación del Jefe de Familia o Grupo. 1 si es educación superior o 0 de cualquier otra forma.

- PREO: Interés por mejorar el bien ambiental. 1 si es así, 0 de cualquier forma diferente.

- OCUP: Ocupación. Si pertenece a la clase de dependencia ( $=1$ ) y 0 en caso contrario.

Los siguientes modelos incrementales de utilidad se basaron en (HANEMANN, 1984) (DUCCI, 1988) y (ARDILA, 1993). 


\section{MATERIALES Y EQUIPOS UTILIZADOS}

Los materiales y equipos necesarios que fueron utilizados en el trabajo de campo son los siguientes:

- Encuestas

- Cámara fotográfica

- GPS

- Sonómetro
- Escala de Ringelmann

- Cinta métrica

- Computador portátil

- Cartas Topográficas

- Botas de caucho

- Poncho de agua

- Guantes

\section{RESULTADOS Y DISCUSIÓN}

Entre los principales bienes y servicios ambientales que presta la Reserva Ecológica, se determinó un total de USD \$13.371.919,03 dólares, proporcionados por el cálculo de los diferentes recursos valorados, en función de la alta disponi- bilidad de información y métodos de valoración aplicados de acuerdo con la metodología compuesta para la valoración económica de los bienes y servicios ambientales en la Reserva Ecológica "Hola Vida".

\section{Tabla 1. Valoración económica tołal de los servicios ambientales de la Reserva Ecológica "Hola Vida"}

\begin{tabular}{|c|c|}
\hline $\begin{array}{l}\text { SERVICIO AMBIENTAL VALORADO DENTRO DE LA } \\
\text { RESERVA ECOLÓGICA HOLA VIDA }\end{array}$ & $\begin{array}{c}\text { VALOR } \\
\text { ESTIMADO\$ }\end{array}$ \\
\hline $\begin{array}{l}\text { Valor estimado de volumen de madera existente en } \\
\text { la Reserva Ecológica }\end{array}$ & $10 \cdot 889.719,99$ \\
\hline Valor del recurso hidrico (año) & $2 * 154.224,16$ \\
\hline \multicolumn{2}{|l|}{ Valoración costo de oportunidad } \\
\hline Artesanias (año) & $1.800,00$ \\
\hline Alimentación (año) & $12.775,00$ \\
\hline Hospedaje (año) & $9.125,00$ \\
\hline \multicolumn{2}{|l|}{ Costo de viaje } \\
\hline Ingreso de turistas (año) & $5.400,00$ \\
\hline Almacenamiento de Carbono & $298.874,88$ \\
\hline VALORACIÓN ECONÓMICA TOTAL & $13.371 .919,03$ \\
\hline
\end{tabular}

Elaborado por José Luis Gavidia, 2015 
Luego de valorar los principales bienes y servicios ambientales identificados en la reserva, se pudo determinar que el volumen de madera existente representa el $81,44 \%$ de los ingresos totales calculados; mientras que el recurso hídrico es el 16,11\%; seguido por el almacenamiento de carbono con el 2,24\% y los otros servicios valorados representando el 0,22\%.

A partir de estos resultados, se debe considerar que el recurso hídrico y almacenamiento de carbono, generan en el largo plazo mayores beneficios económicos ya que, el recurso de volumen de madera existente en la reserva, únicamente se podría aprovechar una sola vez y se tendría que reforestar para su uso consecuente. Sin embargo, es necesario resaltar que su regeneración llevaría años, en tal sentido, se considera un recurso finito y de consumo limitado.

El carbono acumulado en la Reserva Ecológica "Hola Vida", es de 166,04 ton/ha promedio, considerando que es un bosque secundario homogéneo. Los beneficios económicos que presenta el servicio ambiental de captura de carbono y la conservación del bosque en la Reserva Ecológica "Hola Vida", permitiría la preservación e incremento de la biodiversidad existente y la reducción de las emisiones de $\mathrm{CO}_{2}$.

Tabla 2. Reportes de estimaciones de biomasa aérea y carbono, en el trópico

\begin{tabular}{|c|c|c|c|c|}
\hline Tipo de bosque & $\begin{array}{c}\text { Biomas } \\
\text { (tha) }\end{array}$ & $\begin{array}{c}\text { Carbono } \\
\text { (t/ha) }\end{array}$ & Fuente & \\
\hline Tropical de Montano* & 290 & 145 & Adams, 1997 & \\
\hline Tropical premontano húmedo* & 284 & 142 & Golley, et al., 1975 & \\
\hline Tropical montano húmedo* & 428 & 214 & Brun, 1976 & \\
\hline $\begin{array}{l}\text { Tropical premontano muy } \\
\text { húmedo* }\end{array}$ & 456 & 228 & $\begin{array}{l}\text { Greenland and } \\
1960\end{array}$ & Kowal, \\
\hline Tropical Húmedo (América)* & 546 & 273 & Folster. 1989 & \\
\hline Tropical Secundario (América) & 310 & 155 & Folster, 1989 & \\
\hline Subtropical secundario (Ecuador) & 255 & 128 & Fehse et al., 1999 & \\
\hline Tropical húmedo cerrado* & 192 & 96 & Brown et al., 1989 & \\
\hline Tropical Húmedo* (América) & 340 & 170 & Brown et al., 1989 & \\
\hline Bosque secundario (Ecuador) & 223 & 112 & López et al., 2002 & \\
\hline Bosque secundario (Ecuador) & 332 & 166 & Gavidia, 2015 & \\
\hline
\end{tabular}

Fuente: (López, Koning, Paredes, \& Benítez, 2002), Modificado por José Luis Gavidia, 2015

La valoración económica de los bienes y servicios ambientales es una herramienta esencial, que permitirá demostrar la importancia de los ecosistemas en el desarrollo de la vida; debido a ello, es necesario utilizar un denominador común que es el dinero, de modo que facilite la toma de decisiones de carácter político en base a fundamentos más amplios. 


\section{MODELO}

\section{Disposición a pagar-DAPag 1}

Last updated: 12/12/15 - 10:35

Modified: $1300 / /$ dap2 $=-(c(1)+c(3) *$ ing $) / c(2)$

\section{Tabla 2. MODELO INCREMENTAL 2 - DISTRIBUCIÓN LOGIT PRSI, PH, ING}

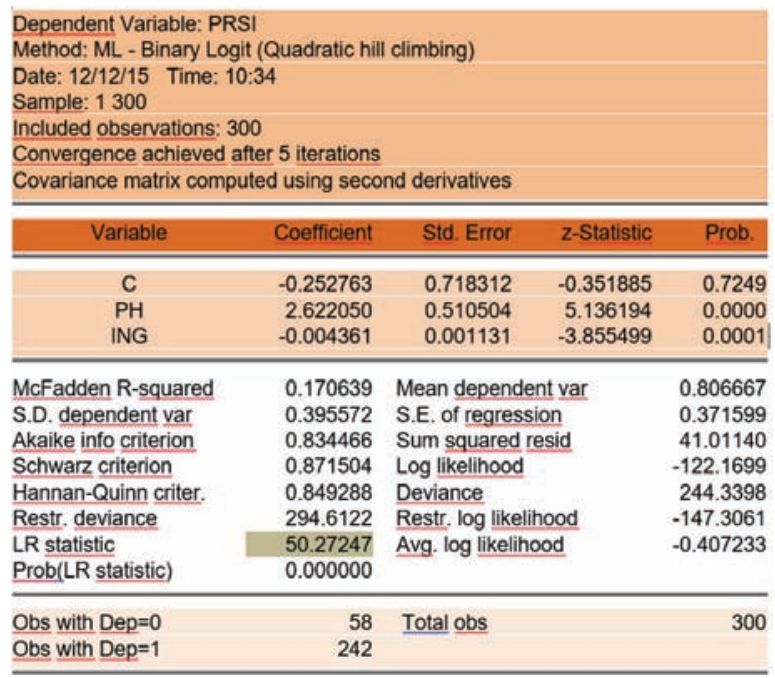

Elaborado por José Luis Gavidia, 2015

El resultado del estudio señala que la población encuestada apoya a la conservación de un entorno natural y paisajístico como es la Reserva Ecológica "Hola Vida"; de los 300 encuestados el $81 \%$ apoya su conservación y estaría de acuerdo en realizar un pago mensual para conservar el recurso y el $19 \%$ no está de acuerdo con pagar un valor para su conservación, pero si les gustaría que se conserve el lugar.
El método empleado para el presente análisis es la Valoración Contingente, que permite conocer las preferencias de los consumidores respecto de determinado bien ambiental y así obtener un valor económico, que para el presente caso determina una disponibilidad de pago de USD \$0,87 ctv. de dólar por individuo de acuerdo con el análisis realizado en DAPag 1, que es el valor medio como disponibili- 
dad a pagar por disfrutar de los beneficios potenciales de los servicios que ofrece la Reserva Ecológica "Hola Vida".

Al presente estudio se ha incluido un Plan de Manejo Ambiental, por cuanto es un instrumento de planificación, mediante el cual se proporciona una guía de medidas, prácticas y acciones e indicadores orientados a aprovechar de manera sostenible los recursos naturales dentro de la Reserva.

\section{CONCLUSIONES}

Los servicios ambientales proporcionados por la Reserva Ecológica "Hola Vida" están en función de su dinámica natural, por cuanto está conformada por un conjunto de bosques naturales y fuentes hídricas que proporcionan innumerables beneficios no solo desde el punto de vista económico para la población local, sino también, desde el punto de vista ecológico como regulador de gases efecto invernadero, almacenamiento de carbono, entre otros.

La valoración de los servicios ambientales se ha convertido en una herramienta estratégica que permitirá direccionar la gestión y manejo sostenible de los recursos naturales dentro de la Reserva.

Entre los principales bienes y servicios ambientales que presta la Reserva Ecológica, se determinó un total de USD \$13.371.919,03 dólares, proporcionados por el cálculo de los diferentes recursos valorados, en función de la alta disponibilidad de información y métodos de valoración aplicados de acuerdo con la metodología compuesta para la valoración económica de los bienes y servicios ambientales en la Reserva Ecológica "Hola Vida".

Los beneficios económicos que presenta el servicio ambiental de captura de carbono y la conservación del bosque en la Reserva Ecológica "Hola Vida", permitiría la preservación e incremento de la biodiversidad existente y la reducción de las emisiones de $\mathrm{CO}_{2}$.

La valoración económica de los bienes y servicios ambientales es una herramienta esencial, que permitirá demostrar la importancia de los ecosistemas en el desarrollo de la vida; debido a ello, es necesario utilizar un denominador común que es el dinero, de modo que facilite la toma de decisiones de carácter político en base a fundamentos más amplios.

El resultado del estudio señala que la población encuestada apoya a la conservación de un entorno natural y paisajístico como es la Reserva Ecológica 
"Hola Vida", de los 300 encuestados el $81 \%$ apoya su conservación y estaría de acuerdo en realizar un pago mensual para conservar el recurso y el 19\% no está de acuerdo con pagar un valor para su conservación pero sí les gustaría que se conserve el lugar, el método empleado para el presente análisis es la Valoración Contingente, que permite conocer las preferencias de los consumidores res- pecto a determinado bien ambiental y así obtener un valor económico, que para el presente caso determina una disponibilidad de pago de USD \$ 0,87 ctv. dólar por individuo de acuerdo con el análisis realizado en DAPag 1, que es el valor medio como disponibilidad a pagar por disfrutar de los beneficios potenciales de los servicios que ofrece la Reserva Ecológica Hola Vida. 


\section{BIBLIOGRAFÍA}

Alpízar, F. (2004). Curso Valoración económica del ambiente. Tema1: Introducción y medidas del bienestar. Costa Rica: CATIE. p. 11-13.

ARDILA, S. (1993). Guía para la utilización de modelos econométricos en aplicaciones del método de valoración contingente. BID, diciembre, 1-24.

DUCCI, J. (1988). Metodología de cuantificación de beneficios. Saneamiento ambiental de Montevideo, Uruguay. Anexo Técnico.

HANEMANN, W. M. (1984). Welfare evaluations in contingent valuation experiments with discrete responses. Amer. J. of Agr. Econ. 66(1), 332-341.

López, M., Koning, F. d., Paredes, H., \& Benítez, P. (2002). Estimación de carbono en biomasa de bosques secundarios y plantaciones forestales en el Noroccidente de Ecuador. Investigación de bosques tropicales. Quito, Ecuador: Institute of soil Science and Forest.

McRoberts, R., Tomppo, E., \& http:, R. C. (8 de octubre de 2015). Diseños de muestreo de las evaluaciones forestales nacionales. Obtenido de Diseños de muestreo de las evaluaciones forestales nacionales.: http://www.fao.org/fileadmin/ user_upload/national_forest_assessment/images/PDFs/Spanish/KR2_ES_ _4_.pdf
Ortiz, E., \& Carrera, F. (2002). Inventarios forestales para bosques latifoliados en América Central. Turialba Costa Rica: Centro agronómico tropical de investigación y enseñanza.

Pascó-Font, A. (29 de julio de 1997). Valoración de los recursos naturales y políticas para la promoción del desarrollo sostenible de la Amazonía (en línea). Obtenido de http://www.idrc.ca/library/ document/101488

Uclés, D. (2006). El valor económico del medio ambiente. España 6p.

UICN. (2012). Un análisis del impacto de las resoluciones de la UICN en los esfuerzos internacionales de la conservación. Unión internacional para la conservación de la naturaleza, 12.

WINDEVOXHEL. (1992). Valoración económica parcial de los manglares de la región II de Nicaragua. Citado en la tesis Valoración económica de bienes y servicios ambientales en sistema agrícolas de San Migue, Petén, Guatemala. Costa Rica: Tesis Mag. Sc CATIE Turrialba. 